\title{
Combining the Pharmacophore Features of Coumarins and 1,4-Substituted 1,2,3-Triazoles to Design New Acetylcholinesterase Inhibitors: Fast and Easy Generation of 4-Methylcoumarins/1,2,3-triazoles Conjugates via Click Chemistry
}

\author{
Fernando C. Torres, ${ }^{a, b}$ Guilherme A. Gonçalves, ${ }^{a}$ Kenia L. Vanzolini, ${ }^{c}$ Aloir A. Merlo, ${ }^{d}$ Bruna Gauer, ${ }^{a}$ \\ Maribete Holzschuh, ${ }^{a}$ Saulo Andrade,${ }^{a}$ Maristela Piedade, ${ }^{a}$ Solange C. Garcia, ${ }^{a}$ Ivone Carvalho, ${ }^{e}$ \\ Gilsane L. von Poser, ${ }^{a}$ Daniel F. Kawano, ${ }^{*, f g}$ Vera L. Eifler-Lima*,a and Quezia B. Cass ${ }^{c}$ \\ ${ }^{a}$ Faculdade de Farmácia, Universidade Federal do Rio Grande do Sul, 90610-000 Porto Alegre-RS, Brazil \\ ${ }^{b}$ Faculdade de Farmácia, Centro Universitário Ritter dos Reis, 91849-440 Porto Alegre-RS, Brazil \\ 'Departamento de Química, Universidade Federal de São Carlos, 13565-905 São Carlos-SP, Brazil \\ ${ }^{d}$ Instituto de Química, Universidade Federal do Rio Grande do Sul, 91501-970 Porto Alegre-RS, Brazil \\ ${ }^{e}$ Faculdade de Ciências Farmacêuticas de Ribeirão Preto, Universidade de São Paulo, \\ 14040-903 Ribeirão Preto-SP, Brazil
}

${ }^{f}$ Faculdade de Ciências Farmacêuticas, Universidade Estadual de Campinas, 13083-859 Campinas-SP, Brazil

${ }^{8}$ Departamento de Química Orgânica, Instituto de Química, Universidade Estadual de Campinas, 13083-970 Campinas-SP, Brazil

\begin{abstract}
Coumarins are a large class of compounds that display a range of interesting biological properties, being considered privileged structures because of the ability of their $2 \mathrm{H}$-chromen-2-one nuclei to bind to multiple pharmacological targets. We hypothesized that the linkage of a second pharmacophore nucleus to the $2 \mathrm{H}$-chromen-2-one core, the 1,2,3-triazole moiety, would entail more selective and pharmacologically active coumarins. Therefore, we describe the synthesis of fourteen 4-methylcoumarins/1,4-substituted 1,2,3-triazole conjugates, which were predicted by in silico methods to inhibit acetylcholinesterase (AChE) and proved to be moderate in vitro inhibitors of this enzyme. Molecular docking simulations suggest that the most active of these compounds has a putative binding mode similar to donepezil, both occupying the peripheral anionic site of AChE, which is associated with the secondary noncholinergic functions of the enzyme. This highlights the potential of this series for further optimization in the search of new coumarins for the treatment of Alzheimer's disease.
\end{abstract}

Keywords: Alzheimer's disease, acetylcholinesterase, coumarins, click chemistry

\section{Introduction}

Coumarins are a large class of compounds that display a wide range of interesting biological properties such as anticoagulant, ${ }^{1}$ antineurodegenerative, ${ }^{2}$ antioxidant, ${ }^{3}$ anticancer ${ }^{4,5}$ and antimicrobial activities. ${ }^{6}$ They are considered good examples of privileged structures, usually rigid, polycyclic heteroatomic systems that are capable to bind to multiple pharmacological targets, thus providing a viable starting point in the search for new drugs. ${ }^{7}$

*e-mail: veraeifler@ufrgs.br; daniel.kawano@fcf.unicamp.br
The $2 \mathrm{H}$-chromen-2-one nucleus is the structural feature that is responsible for the privileged pharmacological actions of coumarins. This planar ring system is composed by one aromatic ring (Figure 1, ring A), capable of establishing hydrophobic, $\pi-\pi, \mathrm{CH}-\pi$ and cation- $\pi$ interactions, and one lactone ring (Figure 1, ring B), which contains two oxygen atoms that may interact via hydrogen bonding with a series of amino acid residues, such as serine, threonine, cysteine, asparagine, glutamine and tyrosine. The $\mathrm{C}-\mathrm{C}$ double bond in the 2-pyrone ring (Figure 1, ring $\mathrm{B}$ ) is also essential for conferring planarity to the $2 H$-chromen-2-one core, allowing charge delocalization between the carbonyl group and the aromatic ring. ${ }^{8}$ 


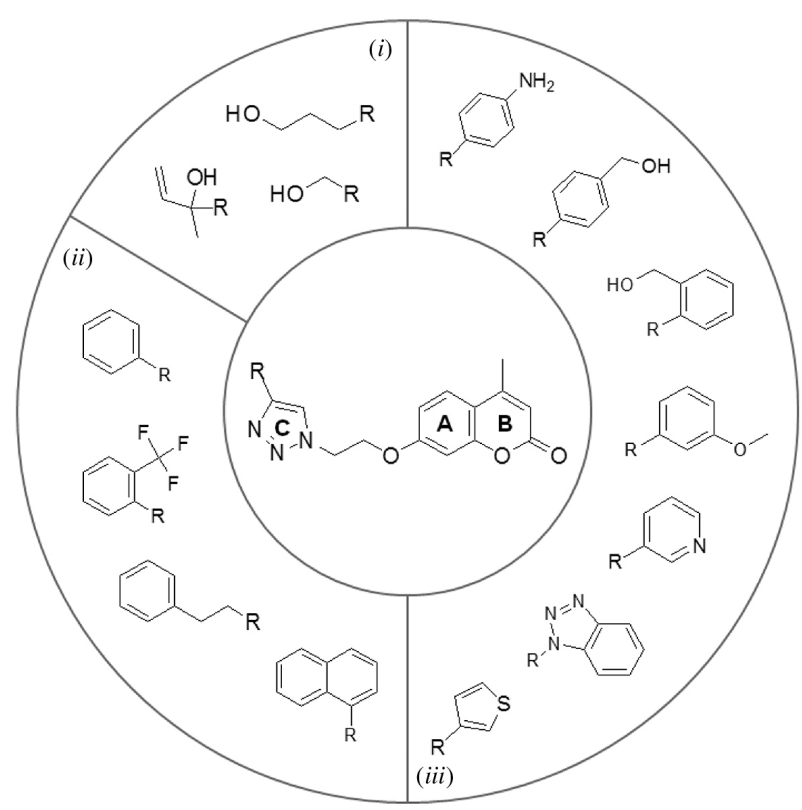

Figure 1. General structure of the 4-methylcoumarins/1,4-substituted 1,2,3-triazole conjugates. The $2 \mathrm{H}$-chromen-2-one core of coumarins is composed of one aromatic ring (ring A) and a lactone (ring B). The 1,2,3-triazole moiety is also depicted (ring C). The substituents at position 4 of the triazole (R) are grouped (counterclockwise) according to the main predicted interactions with the pharmacological target: $(i)$ hydrogen bond donors and acceptors; (ii) aromatic/hydrophobic groups; and (iii) aromatic plus hydrogen bond donors/acceptor or exclusively acceptor groups.

Although the synthesis of privileged structures is certainly a helpful strategy to find new lead compounds, this approach may be not so advantageous from the clinical point of view, since the activation of multiple targets by these planar ring systems may also increase the chances of adverse reactions and drug interactions. It was demonstrated that there is a significant trend for reduced aqueous solubility and increased inhibition of CYP3A4 for planar aromatic compounds, e.g., warfarin, being the inhibition of cytochrome P450 function associated with a higher risk of compound toxicity and severe drug-drug interactions. ${ }^{9}$

Therefore, we hypothesized that the linkage of a second pharmacophore nucleus to the $2 \mathrm{H}$-chromen-2-one core, the 1,2,3-triazole moiety (Figure 1, ring C), would entail more selective and still pharmacologically active coumarins. Triazoles are known to interact with several biological targets via coordination, hydrogen bonds, hydrophobic, $\pi-\pi, \mathrm{CH}-\pi$ and cation- $\pi$ interactions. ${ }^{10}$ Considering that the regioselective synthesis of 1,4-substituted 1,2,3-triazoles is an example of click chemistry, this can also be a good strategy to achieve structural diversity, thus facilitating structure-activity relationship (SAR) studies through the generation of analog libraries. ${ }^{11}$

In this context, herein we report the synthesis of fourteen analogues of hymecromone (7-hydroxy-4-methylcoumarin) in which the 7-hydroxyl group of the coumarin was used to connect, via an ethyl bridge, the $2 H$-chromen-2-one core to 1,4-substituted 1,2,3-triazoles with different substituents at position 4 . These substituents were grouped into three different categories: (i) hydrogen bond donors and acceptors; (ii) aromatic/hydrophobic groups; and (iii) aromatic plus hydrogen bond donors/acceptors or aromatic plus acceptor groups (Figure 1). As predicted by the in silico simulations, most of these analogues proved to be moderate inhibitors of the acetylcholinesterase (AChE), thus highlighting the potential of this series for further optimization in the search of clinically relevant coumarins for the treatment of Alzheimer's disease.

\section{Experimental}

\section{Chemistry}

All chemicals were purchased as reagent grade and used without further purification. Solvents were distilled and/or dried according to standard methods. ${ }^{12}$ Column chromatography was performed on silica gel 60 (0.040-0.063 mm) using dichloromethane $\left(\mathrm{CH}_{2} \mathrm{Cl}_{2}\right)$ and $\mathrm{CH}_{2} \mathrm{Cl}_{2}$ /ethyl acetate (EtOAc), 5:1 or 2:1 (v/v). Microwave-assisted reactions were performed on a CEM Discover ${ }^{\circledR}$ microwave system (Matthews, NC, USA). Melting points were determined on a Fisatom 431 apparatus (São Paulo, SP, Brazil) and are uncorrected. Mass spectrometer (MS) spectra were recorded on Q-Tof micro Waters high resolution mass spectrometer (Milford, MA, USA), operating in electrospray ionization mode. Nuclear magnetic resonance (NMR) spectra were recorded on Bruker Avance 400 DPX (400 MHz) spectrometer (Billerica, MA, USA). Chemical shifts $(\delta)$ are given in parts per million downfield from tetramethylsilane.

\section{7-Hydroxy-4-methylchromen-2-one (3)}

Resorcinol (1 g, $9.1 \mathrm{mmol})$ and ethyl acetoacetate $(1.15 \mathrm{~mL}, 9.1 \mathrm{mmol})$ were added to a round bottom threenecked flask containing concentrated $\mathrm{H}_{2} \mathrm{SO}_{4}(10 \mathrm{~mL})$ in ice bath. The reaction mixture was allowed to stir overnight at room temperature and the total consumption of the reactants was confirmed by thin layer chromatography (TLC; $\mathrm{CH}_{2} \mathrm{Cl}_{2} / \mathrm{EtOAc} 5: 1$, v/v). Ice cold distilled water was then added to precipitate the crude product, which was filtered, dissolved in a 5\% (v/v) aqueous solution of $\mathrm{NaOH}$, filtered again and reprecipitated by the addition of a $10 \%$ (v/v) aqueous solution of $\mathrm{H}_{2} \mathrm{SO}_{4}$ to give $\mathbf{3}$ as a white solid. ${ }^{13}$

Yield 67\%; mp 185-186 ${ }^{\circ} \mathrm{C}$; ${ }^{1} \mathrm{H}$ NMR $(300 \mathrm{MHz}$, DMSO- $\left.d_{6}\right) \delta 10.53(\mathrm{~s}, 1 \mathrm{H}), 7.59(\mathrm{~d}, 1 \mathrm{H}, J 8.7 \mathrm{~Hz}), 6.80$ (dd, $1 \mathrm{H}, J$ 8.7, $2.4 \mathrm{~Hz}), 6.70$ (d, 1H, J 2.4 Hz), $6.13(\mathrm{~d}, 1 \mathrm{H}$, 
$J 1.2 \mathrm{~Hz}), 2.36$ (bs, 3H); ${ }^{13} \mathrm{C}$ NMR (75 MHz, DMSO- $d_{6}$ ) $\delta 161.15,160.30,154.83,153.57,126.64,112.86,112.02$, $110.25,102.17,18.13$; HRMS (ESI) $\mathrm{m} / z$ calcd. for $\mathrm{C}_{10} \mathrm{H}_{8} \mathrm{O}_{3}$ $[\mathrm{M}+\mathrm{H}]^{+}$: 177.0552; found: 177.0553 .

\section{7-(2-Azidoethoxy)-4-methyl-2H-chromen-2-one (5)}

To a solution of 3 (704 mg, $4 \mathrm{mmol}$ ) in $\mathrm{CH}_{3} \mathrm{CN}(30 \mathrm{~mL})$, $\mathrm{K}_{2} \mathrm{CO}_{3}(3.32 \mathrm{~g}, 24 \mathrm{mmol})$ was added and the mixture was allowed to reflux for $15 \mathrm{~min}$. 1,2-Dibromoethane $(1.03 \mathrm{~mL}$, $12 \mathrm{mmol}$ ) was then added and the reaction mixture was refluxed for additional $6 \mathrm{~h}$. Consumption of the reactant was followed by TLC $\left(\mathrm{CH}_{2} \mathrm{Cl}_{2}\right)$ and $\mathrm{K}_{2} \mathrm{CO}_{3}$ was removed by filtration. The crude product was concentrated, dissolved in $\mathrm{CH}_{2} \mathrm{Cl}_{2}$, washed with water, brine and dried over anhydrous $\mathrm{Na}_{2} \mathrm{SO}_{4}$. The residue was purified by column chromatography with a hexane/ethyl acetate gradient to give 7-(2-bromoethoxy)-4-methyl-2 $\mathrm{H}$-chromen-2-one $\mathbf{4}$ as a white solid, which was directly used in the subsequent step.

To a solution of $4(1.7 \mathrm{~g}, 6 \mathrm{mmol})$ in acetone $(30 \mathrm{~mL})$, an aqueous solution of sodium azide $(12 \mathrm{mmol}, 0.78 \mathrm{~g}$ in $5 \mathrm{~mL}$ of $\mathrm{H}_{2} \mathrm{O}$ ) was added. The mixture was refluxed for $12 \mathrm{~h}$ and the reaction followed by TLC $\left(\mathrm{CH}_{2} \mathrm{Cl}_{2}\right)$. The mixture was concentrated and the residue extracted with ethyl acetate $(3 \times 30 \mathrm{~mL})$. The organic phase was washed with brine, dried over anhydrous $\mathrm{Na}_{2} \mathrm{SO}_{4}$ and concentrated to afford $\mathbf{5}$ as a white solid. ${ }^{14}$

Yield 89\%; mp 101-102 ${ }^{\circ} \mathrm{C} ;{ }^{1} \mathrm{H}$ NMR $(300 \mathrm{MHz}$, DMSO- $\left.d_{6}\right) \delta 7.70(\mathrm{~d}, 1 \mathrm{H}, J 8.9 \mathrm{~Hz}), 7.02(\mathrm{~d}, 1 \mathrm{H}, J 2.7 \mathrm{~Hz})$, 6.98 (dd, 1H, J 8.9, $2.7 \mathrm{~Hz}), 6.23$ (d, 1H, J 1.2 Hz), 4.29 (t, $2 \mathrm{H}, J 4.8 \mathrm{~Hz}$ ), 3.70 (t, 2H, J 4.8 Hz), 2.40 (d, 3H, J $1.2 \mathrm{~Hz}$ ); ${ }^{13} \mathrm{C}$ NMR (75 MHz, DMSO- $\left.d_{6}\right) \delta 160.97,160.09,154.67$, 153.39, 126.60, 113.45, 112.46, 111.37, 101.29, 67.42, 49.38, 18.15; HRMS (ESI) $\mathrm{m} / z$ calcd. for $\mathrm{C}_{12} \mathrm{H}_{12} \mathrm{~N}_{3} \mathrm{O}_{3}$ $[\mathrm{M}+\mathrm{H}]^{+}:$: 246.0879; found: 246.0879 .

\section{General procedure for the preparation of compounds $7 \mathbf{a}-\mathbf{7 n}$}

Acetylene derivatives 6a-6n $\left(0.15 \mathrm{mmol} \mathrm{L}^{-1}\right.$, 1.1 equiv) were added to a solution of compound 5 in dimethylformamide (DMF; $0.5-1 \mathrm{~mol} \mathrm{~L}^{-1}, 0.1 \mathrm{~mL}$ ) in a microwave flask $(0.2 \mathrm{~mL})$ equipped with a stirring bar. Sodium ascorbate ( 0.1 equiv) and $\mathrm{CuSO}_{4}(0.03$ equiv) were added, the tube was sealed and the mixture was stirred for $25 \mathrm{~s}$ at room temperature followed by heating under microwave irradiation at $70{ }^{\circ} \mathrm{C}(18 \mathrm{~W})$ in cycles of $20 \mathrm{~min}$. Consumption of the reactant was followed by TLC $\left(\mathrm{CH}_{2} \mathrm{Cl}_{2} /\right.$ EtOAc $\left.2: 1, \mathrm{v} / \mathrm{v}\right)$. The reaction mixture was partitioned between $\mathrm{H}_{2} \mathrm{O}$ and EtOAc, the aqueous phase was extracted with EtOAc ( 3 to 5 times depending on the solubility of the compound). The organic phase was dried over $\mathrm{MgSO}_{4}$, filtered, concentrated and the residue purified by column chromatography with a $\mathrm{CH}_{2} \mathrm{Cl}_{2} / \mathrm{EtOAc}$ gradient. ${ }^{15}$

7-(2-(4-(Hydroxymethyl)-1H-1,2,3-triazol-1-yl)ethoxy)-4methyl-2H-chromen-2-one (7a)

White solid; yield 78\%; mp 164-165 ${ }^{\circ} \mathrm{C}$; ${ }^{1} \mathrm{H}$ NMR $\left(300 \mathrm{MHz}\right.$, DMSO- $\left.d_{6}\right) \delta 8.05(\mathrm{~s}, 1 \mathrm{H}), 7.67(\mathrm{~d}, 1 \mathrm{H}, J 8.7 \mathrm{~Hz})$, $7.01(\mathrm{~d}, 1 \mathrm{H}, J 2.4 \mathrm{~Hz}), 6.94(\mathrm{dd}, 1 \mathrm{H}, J 8.7,2.4 \mathrm{~Hz}), 6.21$ (d, $1 \mathrm{H}, J 1.1 \mathrm{~Hz}), 5.17(\mathrm{t}, 1 \mathrm{H}, J 5.4 \mathrm{~Hz}), 4.78(\mathrm{t}, 2 \mathrm{H}, J 5.1 \mathrm{~Hz})$, 4.54-4.51 (m, 4H), $2.38(\mathrm{~d}, 3 \mathrm{H}, J 1.1 \mathrm{~Hz}) ;{ }^{13} \mathrm{C}$ NMR (75 MHz, DMSO- $\left.d_{6}\right) \delta 160.8,160.0,154.6,153.3,148.0$, 126.5, 123.1, 113.5, 112.4, 111.4, 101.4, 66.8, 55.0, 48.6, 18.1; HRMS (ESI) $m / z$ calcd. for $\mathrm{C}_{15} \mathrm{H}_{15} \mathrm{~N}_{3} \mathrm{O}_{4}[\mathrm{M}+\mathrm{H}]^{+}$: 302.1141; found: 302.1146 .

7-(2-(4-(3-Methyl-1-penten-3-ol)-1H-1,2,3-triazol-1-yl) ethoxy)-4-methyl-2H-chromen-2-one (7b)

White solid; yield 65\%; $\mathrm{mp} 134{ }^{\circ} \mathrm{C} ;{ }^{1} \mathrm{H}$ NMR $(400 \mathrm{MHz}$, DMSO- $d_{6}$ ) $\delta 7.93(\mathrm{~s}, 1 \mathrm{H}), 7.67$ (d, $\left.1 \mathrm{H}, J 8.8 \mathrm{~Hz}\right), 6.99$ (d, $1 \mathrm{H}, J 2.6 \mathrm{~Hz}), 6.93(\mathrm{dd}, 1 \mathrm{H}, J$ 8.8, $2.6 \mathrm{~Hz}), 6.22(\mathrm{~d}, 1 \mathrm{H}$, $J 1.2 \mathrm{~Hz}), 6.14(\mathrm{dd}, 1 \mathrm{H}, J 17.2,10.6 \mathrm{~Hz}), 5.41(\mathrm{~s}, 1 \mathrm{H}), 5.16$ (dd, $1 \mathrm{H}, J 17.2,1.8 \mathrm{~Hz}$ ), 4.97 (dd, $1 \mathrm{H}, J 10.6,1.8 \mathrm{~Hz}), 4.76$ (t, 2H, J 5.0 Hz), $4.53(\mathrm{t}, 2 \mathrm{H}, J 5.0 \mathrm{~Hz}), 2.38(\mathrm{~s}, 3 \mathrm{H}), 1.52$ (bs, $3 \mathrm{H}) ;{ }^{13} \mathrm{C}$ NMR (100 MHz, DMSO- $\left.d_{6}\right) \delta 160.8,160.0$, 154.6, 153.8, 153.3, 145.0, 140.7, 126.5, 121.7, 113.5, 112.4, 111.4, 111.1, 101.4, 69.6, 66.8, 48.6, 28.6, 18.1; HRMS (ESI) $m / z$ calcd. for $\mathrm{C}_{18} \mathrm{H}_{19} \mathrm{~N}_{3} \mathrm{O}_{4}[\mathrm{M}+\mathrm{Na}+\mathrm{H}]^{+}$: 365.1352; found: 365.1358 .

7-(2-(4-(4-Propan-1-ol)-1H-1,2,3-triazol-1-yl)ethoxy)-4methyl-2H-chromen-2-one (7c)

White solid; yield $80 \%$; mp $149{ }^{\circ} \mathrm{C} ;{ }^{1} \mathrm{H}$ NMR $(400 \mathrm{MHz}$, DMSO- $\left.d_{6}\right) \delta 7.92(\mathrm{~s}, 1 \mathrm{H}), 7.67(\mathrm{~d}, 1 \mathrm{H}, J 8.8 \mathrm{~Hz}), 6.99(\mathrm{~d}$, $1 \mathrm{H}, J 2.4 \mathrm{~Hz}), 6.94(\mathrm{dd}, 1 \mathrm{H}, J$ 8.8, $2.4 \mathrm{~Hz}), 6.21(\mathrm{~d}, 1 \mathrm{H}$, $J 1.2 \mathrm{~Hz}), 4.74(\mathrm{t}, 2 \mathrm{H}, J 4.8 \mathrm{~Hz}), 4.51(\mathrm{t}, 2 \mathrm{H}, J 4.8 \mathrm{~Hz})$, $3.42(\mathrm{t}, 2 \mathrm{H}, J 6.4 \mathrm{~Hz}), 2.63(\mathrm{t}, 2 \mathrm{H}, J 7.6 \mathrm{~Hz}), 2.38(\mathrm{~s}, 3 \mathrm{H})$, $1.72(\mathrm{~m}, 2 \mathrm{H}) ;{ }^{13} \mathrm{C}$ NMR $\left(100 \mathrm{MHz}\right.$, DMSO- $\left.d_{6}\right) \delta 160.8$, 160.0, 154.6, 153.3, 146.8, 140.7, 126.5, 122.3, 113.5, 112.4, 111.4, 101.4, 66.8, 60.0, 48.6, 32.3, 21.6, 18.1; HRMS (ESI) $m / z$ calcd. for $\mathrm{C}_{17} \mathrm{H}_{19} \mathrm{~N}_{3} \mathrm{O}_{4}[\mathrm{M}+\mathrm{H}]^{+}: 330.1454$; found: 330.1443 .

7-(2-(4-Phenyl-1H-1,2,3-triazol-1-yl)ethoxy)-4-methyl-2Hchromen-2-one $\mathbf{( 7 d )}$

White solid; yield 91\%; mp $180{ }^{\circ} \mathrm{C}$; ${ }^{1} \mathrm{H}$ NMR $\left(300 \mathrm{MHz}\right.$, DMSO- $\left.d_{6}\right) \delta 8.66(\mathrm{~s}, 1 \mathrm{H}), 7.85(\mathrm{~m}, 2 \mathrm{H}), 7.67(\mathrm{~d}$, $1 \mathrm{H}, J 8.9 \mathrm{~Hz}), 7.44(\mathrm{t}, 2 \mathrm{H}, J 7.2 \mathrm{~Hz}), 7.33(\mathrm{t}, 1 \mathrm{H}, J 7.2 \mathrm{~Hz})$, $7.04(\mathrm{~d}, 1 \mathrm{H}, J 2.4 \mathrm{~Hz}), 6.97$ (dd, 1H, J 8.9, $2.4 \mathrm{~Hz}), 6.20$ (d, $1 \mathrm{H}, J 0.9 \mathrm{~Hz}), 4.85(\mathrm{t}, 2 \mathrm{H}, J 5.0 \mathrm{~Hz}), 4.58(\mathrm{t}, 2 \mathrm{H}, J 5.0 \mathrm{~Hz})$, 2.37 (d, 3H, $J 0.9 \mathrm{~Hz}) ;{ }^{13} \mathrm{C}$ NMR (75 MHz, DMSO- $d_{6}$ ) 
$\delta 160.8,160.0,154.6,153.3,146.4,130.7,128.9,127.8$, $126.5,125.1,121.9,113.5,112.4,111.4,101.5,66.7,49.0$ 18.1; HRMS (ESI) $\mathrm{m} / z$ calcd. for $\mathrm{C}_{20} \mathrm{H}_{17} \mathrm{~N}_{3} \mathrm{O}_{3}[\mathrm{M}+\mathrm{H}]^{+}$: 348.1348; found: 348.1365 .

7-(2-(4-(2-(Trifluoromethyl)phenyl)-1H-1,2,3-triazol-1-yl) ethoxy)-4-methyl-2H-chromen-2-one (7e)

White solid; yield $85 \%$; mp $157{ }^{\circ} \mathrm{C}$; ${ }^{1} \mathrm{H}$ NMR $(300 \mathrm{MHz}$, DMSO- $\left.d_{6}\right) \delta 8.40(\mathrm{~d}, 1 \mathrm{H}, J 0.9 \mathrm{~Hz}), 7.87-7.66(\mathrm{~m}, 6 \mathrm{H}), 7.01$ (d, 1H, J 2.4 Hz), $6.93(\mathrm{dd}, 1 \mathrm{H}, J 8.7,2.4 \mathrm{~Hz}), 6.21(\mathrm{~d}, 1 \mathrm{H}$, $J 1.2 \mathrm{~Hz}), 4.91$ (t, 2H, J $4.8 \mathrm{~Hz}), 4.59$ (t, 2H, J $4.8 \mathrm{~Hz})$, $2.38(\mathrm{~d}, 3 \mathrm{H}, J 1.2 \mathrm{~Hz}) ;{ }^{13} \mathrm{C} \mathrm{NMR}\left(75 \mathrm{MHz}, \mathrm{DMSO}-d_{6}\right)$ $\delta 160.8,160.0,154.6,153.3,143.2,132.6,131.7,129.5$, $128.8,126.5,126.3,126.2,124.6,122.1,113.5,112.4$, 111.4, 101.4, 66.7, 48.9, 18.1; HRMS (ESI) $\mathrm{m} / \mathrm{z}$ calcd. for $\mathrm{C}_{21} \mathrm{H}_{16} \mathrm{~F}_{3} \mathrm{~N}_{3} \mathrm{O}_{3}[\mathrm{M}+\mathrm{H}]^{+}:$416.1222; found: 416.1234 .

7-(2-(4-(Naphthalen-1-yl)-1 H-1,2,3-triazol-1-yl)ethoxy)-4methyl-2H-chromen-2-one (7f)

Dark yellow liquid; yield 59\%; ${ }^{1} \mathrm{H}$ NMR (400 MHz, $\left.\mathrm{CDCl}_{3}\right) \delta 8.33-8.31(\mathrm{~m}, 1 \mathrm{H}), 8.03(\mathrm{~s}, 1 \mathrm{H}), 7.88-7.86(\mathrm{~m}$, 2H), 7.70 (d, 1H, J 6.8 Hz), 7.51-7.27 (m, 4H), 6.83-6.80 $(\mathrm{m}, 2 \mathrm{H}), 6.12(\mathrm{~s}, 1 \mathrm{H}), 4.92(\mathrm{t}, 2 \mathrm{H}, J 4.6 \mathrm{~Hz}), 4.50(\mathrm{t}, 2 \mathrm{H}$, $J 4.6 \mathrm{~Hz}), 2.34(\mathrm{~s}, 3 \mathrm{H}) ;{ }^{13} \mathrm{C} \mathrm{NMR}\left(100 \mathrm{MHz}, \mathrm{CDCl}_{3}\right) \delta 161.0$, 160.6, 155.0, 152.4, 146.9, 133.8, 131.0, 129.0, 128.4, 127.7, 127.2, 126.6, 126.0, 125.8, 125.3, 125.2, 123.8, 114.3, 112.4, 112.0, 101.7, 66.7, 49.5, 18.6; HRMS (ESI) $\mathrm{m} / \mathrm{z}$ calcd. for $\mathrm{C}_{24} \mathrm{H}_{19} \mathrm{~N}_{3} \mathrm{O}_{3}[\mathrm{M}+\mathrm{H}]^{+}$: 398.1505; found: 398.1505 .

7-(2-(4-Phenethyl-1 H-1,2,3-triazol-1-yl)ethyloxy)-4-methyl$2 \mathrm{H}$-chromen-2-one $\mathbf{( 7 g )}$

White solid; yield 84\%; mp $135{ }^{\circ} \mathrm{C} ;{ }^{1} \mathrm{H}$ NMR (400 MHz, DMSO- $\left.d_{6}\right) \delta 7.89$ (s, $\left.1 \mathrm{H}\right), 7.67(\mathrm{~d}, 1 \mathrm{H}, J 8.8 \mathrm{~Hz}), 7.26-7.15$ (m, 5H), $6.98(\mathrm{~d}, 1 \mathrm{H}, J 2.4 \mathrm{~Hz}), 6.92(\mathrm{dd}, 1 \mathrm{H}, J 8.8,2.4 \mathrm{~Hz})$, $6.22(\mathrm{~s}, 1 \mathrm{H}), 4.73$ (t, 2H, J 5.0 Hz), 4.49 (t, 2H, J $5.0 \mathrm{~Hz})$, 2.91 (bs, 4H), 2.39 (s, 3H); ${ }^{13} \mathrm{C} \mathrm{NMR}\left(100 \mathrm{MHz}\right.$, DMSO- $\left.d_{6}\right)$ $\delta 160.8,160.0,154.6,153.3,141.1,140.7,128.3,128.2$, 126.5, 125.8, 122.6, 113.5, 112.4, 111.4, 101.4, 66.8, 48.6, 34.8, 26.9, 18.1; HRMS (ESI) $m / z$ calcd. for $\mathrm{C}_{22} \mathrm{H}_{21} \mathrm{~N}_{3} \mathrm{O}_{3}$ $[\mathrm{M}+\mathrm{H}]^{+}$: 376.1661 ; found: 376.1668 ;

7-(2-(4-(2-(Hydroxymethyl)phenyl)-1H-1,2,3-triazol-1-yl) ethoxy)-4-methyl-2H-chromen-2-one (7h)

White solid; yield 80\%; mp $168-169{ }^{\circ} \mathrm{C} ;{ }^{1} \mathrm{H}$ NMR $\left(300 \mathrm{MHz}, \mathrm{DMSO}-d_{6}\right) \delta 8.51(\mathrm{~s}, 1 \mathrm{H}), 7.76-7.73(\mathrm{~m}, 1 \mathrm{H})$, $7.68(\mathrm{~d}, 1 \mathrm{H}, J 8.8 \mathrm{~Hz}), 7.59-7.56(\mathrm{~m}, 1 \mathrm{H}), 7.37-7.35(\mathrm{~m}$, 2H), 7.04 (d, 1H, J 2.4 Hz), 6.96 (dd, 1H, J 8.8, 2.4 Hz), 6.21 (s, 1H), 4.88 (t, 2H, J 5.0 Hz), 4.61-4.57 (m, 4H), 2.38 (s, $3 \mathrm{H}) ;{ }^{13} \mathrm{C}$ NMR $\left(75 \mathrm{MHz}, \mathrm{DMSO}-d_{6}\right) \delta 160.8,160.1,154.6$, 153.3, 145.1, 139.1, 128.7, 128.2, 128.0, 127.8, 127.1, $126.5,124.1,113.5,112.4,111.4,101.5,66.8,61.6,48.9$,
18.1; HRMS (ESI) $m / z$ calcd. for $\mathrm{C}_{21} \mathrm{H}_{19} \mathrm{~N}_{3} \mathrm{O}_{4}[\mathrm{M}+\mathrm{H}]^{+}$: 378.1454; found: 378.1468 .

7-(2-(4-(4-(Hydroxymethyl)phenyl)-1 H-1,2,3-triazol-1-yl) ethoxy)-4-methyl-2H-chromen-2-one (7i)

White solid; yield 89\%; mp $181{ }^{\circ} \mathrm{C} ;{ }^{1} \mathrm{H}$ NMR $\left(400 \mathrm{MHz}, \mathrm{CDCl}_{3}\right) \delta 7.86(\mathrm{~s}, 1 \mathrm{H}), 7.76(\mathrm{~d}, 2 \mathrm{H}, J 8.8 \mathrm{~Hz})$, 7.49 (d, 1H, J $8.8 \mathrm{~Hz}), 6.97$ (d, 2H, J 8.8 Hz), 6.86-6.82 $(\mathrm{m}, 2 \mathrm{H}), 6.15(\mathrm{~s}, 1 \mathrm{H}), 4.84(\mathrm{t}, 2 \mathrm{H}, J 5.0 \mathrm{~Hz}), 4.47(\mathrm{t}, 2 \mathrm{H}$, $J 5.0 \mathrm{~Hz}), 3.85$ (s, 2H), 2.39 (s, 3H); ${ }^{13} \mathrm{C} \mathrm{NMR}(100 \mathrm{MHz}$, $\left.\mathrm{CDCl}_{3}\right) \delta$ 161.0, 160.6, 159.7, 155.1, 152.3, 147.9, 127.1, 125.8, 123.1, 120.0, 114.4, 114.3, 112.6, 112.0, 101.9, 66.8, 55.3, 49.5, 18.7; HRMS (ESI) $m / z$ calcd. for $\mathrm{C}_{21} \mathrm{H}_{19} \mathrm{~N}_{3} \mathrm{O}_{4}$ $[\mathrm{M}+\mathrm{H}]^{+}$: 401.1352; found: 401.1337 .

7-(2-(4-(3-Methoxyphenyl)-1H-1,2,3-triazol-1-yl)ethoxy)-4methyl-2H-chromen-2-one (7j)

White solid; yield 82\%; mp 164-165 ${ }^{\circ} \mathrm{C}$; ${ }^{1} \mathrm{H}$ NMR $\left(300 \mathrm{MHz}, \mathrm{DMSO}-d_{6}\right) \delta 8.68(\mathrm{~s}, 1 \mathrm{H}), 7.67(\mathrm{~d}, 1 \mathrm{H}, J 9.0 \mathrm{~Hz})$, 7.44-7.41 (m, 2H), $7.35(\mathrm{t}, 1 \mathrm{H}, J 8.0 \mathrm{~Hz}), 7.05(\mathrm{~d}, 1 \mathrm{H}$, $J 2.7 \mathrm{~Hz}), 6.97$ (dd, 1H, 9.0, 2.7 Hz), 6.92-6.88 (m, 1H), $6.21(\mathrm{~d}, 1 \mathrm{H}, J 1.2 \mathrm{~Hz}), 4.85(\mathrm{t}, 2 \mathrm{H}, J 5.0 \mathrm{~Hz}), 4.58(\mathrm{t}, 2 \mathrm{H}$, $J 5.0 \mathrm{~Hz}), 3.81(\mathrm{~s}, 3 \mathrm{H}), 2.37(\mathrm{~d}, 3 \mathrm{H}, J 1.2 \mathrm{~Hz}) ;{ }^{13} \mathrm{C} \mathrm{NMR}$ $\left(75 \mathrm{MHz}, \mathrm{DMSO}-d_{6}\right) \delta 160.83,160.07,159.66,154.62$, $153.36,146.29,132.02,130.05,126.55,122.21,117.50$, $113.53,112.48,111.40,110.39,101.48,66.73,55.11$, 49.04, 18.13; HRMS (ESI) $m / z$ calcd. for $\mathrm{C}_{21} \mathrm{H}_{19} \mathrm{~N}_{3} \mathrm{O}_{4}$ $[\mathrm{M}+\mathrm{H}]^{+}:$378.1454; found: 378.1468 .

7-(2-(4-(4-Aminophenyl)-1 H-1,2,3-triazol-1-yl)ethoxy)-4methyl-2H-chromen-2-one (7k)

White solid; yield 63\%; mp $175{ }^{\circ} \mathrm{C} ;{ }^{1} \mathrm{H}$ NMR $(300 \mathrm{MHz}$, DMSO- $\left.d_{6}\right) \delta 8.35(\mathrm{~s}, 1 \mathrm{H}), 7.67(\mathrm{~d}, 1 \mathrm{H}, J 8.7 \mathrm{~Hz}), 7.49(\mathrm{~d}$, $2 \mathrm{H}, J 8.7 \mathrm{~Hz}), 7.05$ (d, 1H, J 2.4 Hz), 6.97 (dd, 1H, J 8.7, $2.4 \mathrm{~Hz}), 6.60$ (d, 2H, J 8.7 Hz), 6.22 (d, 1H, J1.1 Hz), 5.24 (s, 2H), 4.79 (t, 2H, J 5.0 Hz), 4.55 (t, 2H, J 5.0 Hz), 2.38 (d, $1 \mathrm{H}, J 1.1 \mathrm{~Hz}$ ); HRMS (ESI) $m / z$ calcd. for $\mathrm{C}_{20} \mathrm{H}_{18} \mathrm{~N}_{4} \mathrm{O}_{3}$ $[\mathrm{M}+\mathrm{H}]^{+}:$363.1457; found: 363.1449.

7-(2-(4-(Pyridin-3-yl)-1H-1,2,3-triazol-1-yl)ethoxy)-4-methyl$2 \mathrm{H}$-chromen-2-one (7I)

White solid; yield $72 \%$; mp $174{ }^{\circ} \mathrm{C} ;{ }^{1} \mathrm{H}$ NMR $\left(400 \mathrm{MHz}, \mathrm{DMSO}-d_{6}\right) \delta 9.06(\mathrm{~s}, 1 \mathrm{H}), 8.79(\mathrm{~s}, 1 \mathrm{H}), 8.54(\mathrm{~d}$, $1 \mathrm{H}, J 3.2 \mathrm{~Hz}), 8.23-8.21(\mathrm{~m}, 1 \mathrm{H}), 7.66$ (d, $1 \mathrm{H}, J 8.6 \mathrm{~Hz})$, 7.50-7.46 (m, 1H), 7.05 (d, 1H, J $2.2 \mathrm{~Hz}), 6.98$ (dd, 1H, $J$ 8.6, $2.2 \mathrm{~Hz}), 6.21$ (d, 1H, $0.8 \mathrm{~Hz}), 4.88$ (t, 2H, J 5.0 Hz), $4.58(\mathrm{t}, 2 \mathrm{H}, J 5.0 \mathrm{~Hz}), 2.37$ (d, 3H, J $0.8 \mathrm{~Hz}) ;{ }^{13} \mathrm{C} \mathrm{NMR}$ $\left(100 \mathrm{MHz}, \mathrm{DMSO}-d_{6}\right) \delta 160.8,160.1,154.7,153.4,148.9$, $146.4,143.7,132.4,126.5,124.0,122.7,113.6,112.5$, 111.4, 101.6, 66.8, 49.2, 18.1; HRMS (ESI) $\mathrm{m} / \mathrm{z}$ calcd. for $\mathrm{C}_{19} \mathrm{H}_{16} \mathrm{~N}_{4} \mathrm{O}_{3}[\mathrm{M}+\mathrm{H}]^{+}:$349.1301; found: 349.1291 . 
7-(2-(4-((1H-Benzo[d][1,2,3]triazol-1-yl)methyl)-1H-1,2,3triazol-1-yl)ethoxy)-4-methyl-2H-chromen-2-one (7m)

White solid; yield 85\%; mp 173-174 ${ }^{\circ} \mathrm{C}$; ${ }^{1} \mathrm{H}$ NMR $\left(300 \mathrm{MHz}\right.$, DMSO- $\left.d_{6}\right) \delta 8.35(\mathrm{~s}, 1 \mathrm{H}), 7.95(\mathrm{~d}, 1 \mathrm{H}, J 8.1 \mathrm{~Hz})$, $7.69(\mathrm{~d}, 1 \mathrm{H}, J 9.0 \mathrm{~Hz}), 7.36-7.31(\mathrm{~m}, 3 \mathrm{H}), 6.97(\mathrm{~d}, 1 \mathrm{H}$, $J 2.4 \mathrm{~Hz}), 6.89(\mathrm{dd}, 1 \mathrm{H}, J 9.0,2.4 \mathrm{~Hz}), 6.23(\mathrm{~d}, 1 \mathrm{H}, J 1.1 \mathrm{~Hz})$, $5.68(\mathrm{~s}, 2 \mathrm{H}), 4.78(\mathrm{t}, 2 \mathrm{H}, J 5.0 \mathrm{~Hz}), 4.42(\mathrm{t}, 2 \mathrm{H}, J 5.0 \mathrm{~Hz}), 2.40$ $(\mathrm{d}, 3 \mathrm{H}, J 1.1 \mathrm{~Hz}) ;{ }^{13} \mathrm{C}$ NMR $\left(75 \mathrm{MHz}\right.$, DMSO- $\left.d_{6}\right) \delta 160.7$, 160.1, 154.6, 153.4, 142.5, 139.9, 128.1, 127.5, 127.2, 126.5, 124.7, 119.5, 113.6, 112.4, 111.4, 108.9, 101.4, 72.7, 66.7, 48.9, 18.1; HRMS (ESI) $m / z$ calcd. for $\mathrm{C}_{20} \mathrm{H}_{15} \mathrm{~N}_{6} \mathrm{O}_{4}[\mathrm{M}+\mathrm{H}]^{+}$: 404.1233; found: 404.1213 .

7-(2-(4-(Thiophen-3-yl)-1H-1,2,3-triazol-1-yl)ethoxy)-4methyl-2H-chromen-2-one $(7 \mathbf{n})$

White solid; yield $62 \%$; mp $163{ }^{\circ} \mathrm{C} ;{ }^{1} \mathrm{H}$ NMR $\left(400 \mathrm{MHz}, \mathrm{DMSO}-d_{6}\right) \delta 8.52$ (s, 1H), 7.85 (dd, $1 \mathrm{H}, J 3.0$, $1.2 \mathrm{~Hz}), 7.68-7.63(\mathrm{~m}, 2 \mathrm{H}), 7.52(\mathrm{dd}, 1 \mathrm{H}, J 4.8,1.2 \mathrm{~Hz})$, $7.04(\mathrm{~d}, 1 \mathrm{H}, J 2.8 \mathrm{~Hz}), 6.97(\mathrm{dd}, 1 \mathrm{H}, J 8.8,2.8 \mathrm{~Hz}), 6.21(\mathrm{~d}$, $1 \mathrm{H}, J 1.2 \mathrm{~Hz}), 4.84(\mathrm{t}, 2 \mathrm{H}, J 4.6 \mathrm{~Hz}), 4.56(\mathrm{t}, 2 \mathrm{H}, J 4.6 \mathrm{~Hz})$, $2.37(\mathrm{~d}, 3 \mathrm{H}, J 1.2 \mathrm{~Hz}) ;{ }^{13} \mathrm{C}$ NMR (100 MHz, DMSO- $\left.d_{6}\right)$ $\delta 160.8,160.1,154.6,153.3,143.0,132.0,127.1,126.5$, $125.8,121.7,120.8,113.5,112.5,111.4,101.5,66.8,48.9$, 18.1; HRMS (ESI) $m / z$ calcd. for $\mathrm{C}_{18} \mathrm{H}_{17} \mathrm{~N}_{3} \mathrm{O}_{3} \mathrm{~S}[\mathrm{M}+\mathrm{H}]^{+}$: 355.0991; found: 355.1007 .

Acetylcholinesterase (AChE) screening assay with immobilized capillary enzyme reactor

The screening assay was carried out as previously reported.$^{16}$ Briefly, AChE (E.C. 3.1.1.7) from Electrophorus electricus was immobilized onto the internal surface of an open tubular capillary $(30 \times 0.01 \mathrm{~cm}$ i.d.) producing AChEimmobilized capillary enzyme reactor (ICER). Ammonium acetate solution ( $15 \mathrm{mmol} \mathrm{L}^{-1}, \mathrm{pH}$ 8.0) was used as mobile phase, in a flow rate of $0.05 \mathrm{~mL} \mathrm{~min}^{-1}$. Methanol was used to improve ionization. The total analysis time was $20 \mathrm{~min}$. Assay was carried out monitoring choline's precursor ion $(\mathrm{M})^{+} 104 \mathrm{~m} / \mathrm{z}$ and its fragment $\left(\mathrm{C}_{2} \mathrm{H}_{3} \mathrm{OH}\right)-(\mathrm{M})^{+} 60 \mathrm{~m} / \mathrm{z}$ by multiple-reaction monitoring (MRM) in which the choline precursor ion was used for quantification while MRM ratio and the first transition were used for confirmatory purposes.

The 15 synthesized coumarins were then evaluated for the inhibition of AChE through the hydrolysis of acetycholine (AChE-ICER). To this end, a $1.0 \mathrm{mmol} \mathrm{L}^{-1}$ methanol stock solution was prepared for each tested compound. The $100 \mu \mathrm{L}$ assay samples were prepared with ammonium acetate solution $\left(15.0 \mathrm{mmol} \mathrm{L}^{-1}, \mathrm{pH} 8.0\right)$ containing $25.0 \mu \mathrm{L}$ acetycholine aqueous solution $\left(1960 \mu \mathrm{mol} \mathrm{L}^{-1}\right)$ and $20.0 \mu \mathrm{L}$ of the tested compound methanol stock solution. Analyses were carried out by aliquots injection of $10.0 \mu \mathrm{L}$ of each tested coumarins. For each tested compound, a negative control (absence of acetycholine) and a positive control samples (acetylcholine and absence of coumarin) were analyzed. The percent inhibition for each compound was calculated using equation 1 :

Inhibition $(\%)=100-\left(\frac{\mathrm{P}_{\mathrm{i}}}{\mathrm{P}_{0}} \times 100\right)$

where $\mathrm{P}_{\mathrm{i}}$ stands for the production of choline quantified from the hydrolysis of acetylcholine in the presence of the tested compounds, and $\mathrm{P}_{0}$ for the production of choline as in the positive control experiment.

\section{Molecular modeling studies}

The tridimensional structures of compounds were built using the on-line version of Corina, ${ }^{17}$ which ascribes to 3D structures pre-defined bond lengths and angles depending on the type of bond, type of atom and hybridization state. Corina also defines the most probable torsional angles according to the nature of the structure (acyclic, small/ medium rings, macro/polycyclic, etc.), being able to correctly reproduce a varied number of X-ray structures. ${ }^{18}$ Molecular geometries were then refined using the AM1 semi-empirical force field with the Broyden-FletcherGoldfarb-Shanno (BFGS) algorithm, as implemented in the ArgusLab. ${ }^{19}$ The structure was considered optimized when its conformational energy gradient was below $0.001 \mathrm{kcal} \mathrm{mol}^{-1}$.

Predictions of pharmacological targets were performed using PharmMapper. ${ }^{20}$ PharmMapper identifies potential target candidates for a given small molecule by mapping the pharmacophore groups in multiple conformations of this compound to subsequently search these pharmacophores in a large, in-house repertoire of pharmacophore database, containing more than 7,000 receptor-based pharmacophore models (covering over 1500 drug targets information). ${ }^{21}$ Predictions were performed using the general structure of 4-methylcoumarins/1,4-substituted 1,2,3-triazole conjugates with a methyl substituent at the position 4 of the triazole since all compounds have a carbon atom at this position. Default setting was used for the maximum generated conformations and the compound was screened against all pharmacophore models available in the database.

Molecular docking simulations were performed using the structure of Torpedo californica AChE complexed with an analog of tacrine, 4-amino-5-fluoro-2-methyl3-(3-trifluoroacetylbenzylthiomethyl)quinoline (protein data bank, PDB code 1HBJ). The structure was checked 
for missing atoms and amino acid residues using PDB_HYDRO ${ }^{22}$ but none was identified. Protonation states for the amino acid residues of acetylcholinesterase were calculated at the physiological $\mathrm{pH}$ using PROPKA ${ }^{23}$ and set in GOLD $5.3^{24}$ for the docking simulations.

GOLD is a widely used docking program in the pharmaceutical industry developed by collaboration between the University of Sheffield (Sheffield, UK), GlaxoSmithKline (London, UK) and the Cambridge Crystallographic Data Centre (CCDC). ${ }^{25}$ GOLD uses genetic sampling algorithms, which are inspired by the Darwin's theory of the evolution. The degrees of freedom of the ligand are encoded as binary strings called genes that make up a chromosome, thus, actually representing the pose of the ligand. Therefore, genetic algorithms demand that the fittest individuals (poses) are carried on to the next generation and random or biased mutations can be made to increase genetic (conformational) diversity. ${ }^{26}$

Simulations were performed inside a $10 \AA$ sphere, for which the geometric center ( $\mathrm{x}, \mathrm{y}$ and $\mathrm{z}$ coordinates) was determined by calculating the centroid of 4-amino-5-fluoro2-methyl-3-(3-trifluoroacetylbenzylthiomethyl)quinoline in Discovery Studio Visualizer 4.0. ${ }^{27}$ Default values were set for the genetic algorithm parameters and GoldScore was defined as the score function. Redocking of the crystallographic ligand into the binding site of acetylcholinesterase yielded a heavy atom root mean square deviation (RMSD) of $1.1591 \AA$. The best pose of the compounds was selected for visual inspection and analyses of protein-ligand interactions in Discovery Studio Visualizer.

\section{Results and Discussion}

The synthetic route for the 4-methylcoumarins/1,4substituted 1,2,3-triazole is outlined in Scheme 1:
7 -Hydroxy-4-methylchromen-2-one (3, 4-methylumbelliferone) was synthesized using the Pechmann reaction, ${ }^{13}$ where a phenol 1, under acid catalysis, undergoes electrophilic aromatic substitution with a $\beta$-ketoester 2 to give an intermediate that cyclizes by a transesterification reaction to produce the $2 \mathrm{H}$-chromen-2one nucleus. ${ }^{8}$ Alkylation of the phenolic group of $\mathbf{3}$ using 1,2-dibromoethane and potassium carbonate in acetonitrile yielded 4 , which was then converted into the azide derivative $\mathbf{5}$ via nucleophilic substitution by using sodium azide in acetone. ${ }^{14}$ Synthesis of 4-methylcoumarins/1,4substituted 1,2,3-triazole conjugates was, then, achieved by coupling the azido-coumarin $\mathbf{5}$ with a set of structurally diverse terminal alkynes by using the copper(I)-catalyzed alkyne-azide cycloaddition reaction, ${ }^{15}$ in good yields and with complete regioselectivity.

To predict the most feasible pharmacological target for these compounds, we performed a search using PharmMapper, which maps the pharmacophore groups in small molecules to search for complementary pharmacophores in a drug target database. ${ }^{21}$ Results for the top five predicted targets are shown in Table 1, illustrating that the acetylcholinesterase enzyme displayed the best fit score regarding the complementary between the pharmacophore groups of the ligand with the corresponding functionalities in the macromolecular target.

This theoretical prediction is supported by the fact that several coumarins are known to inhibit the acetylcholinesterase enzyme. ${ }^{28}$ Ensaculin (Figure 2, 8) is probably the most prominent of these compounds, being identified as a lead compound when a series of synthetically modified coumarins were screened. This coumarin demonstrated to have facilitatory effects on learning and memory in rats and to inhibit rat brain acetylcholinesterase activity in vitro with an $\mathrm{IC}_{50}$ of $0.36 \mu \mathrm{mol} \mathrm{L} \mathrm{L}^{-1}$, without

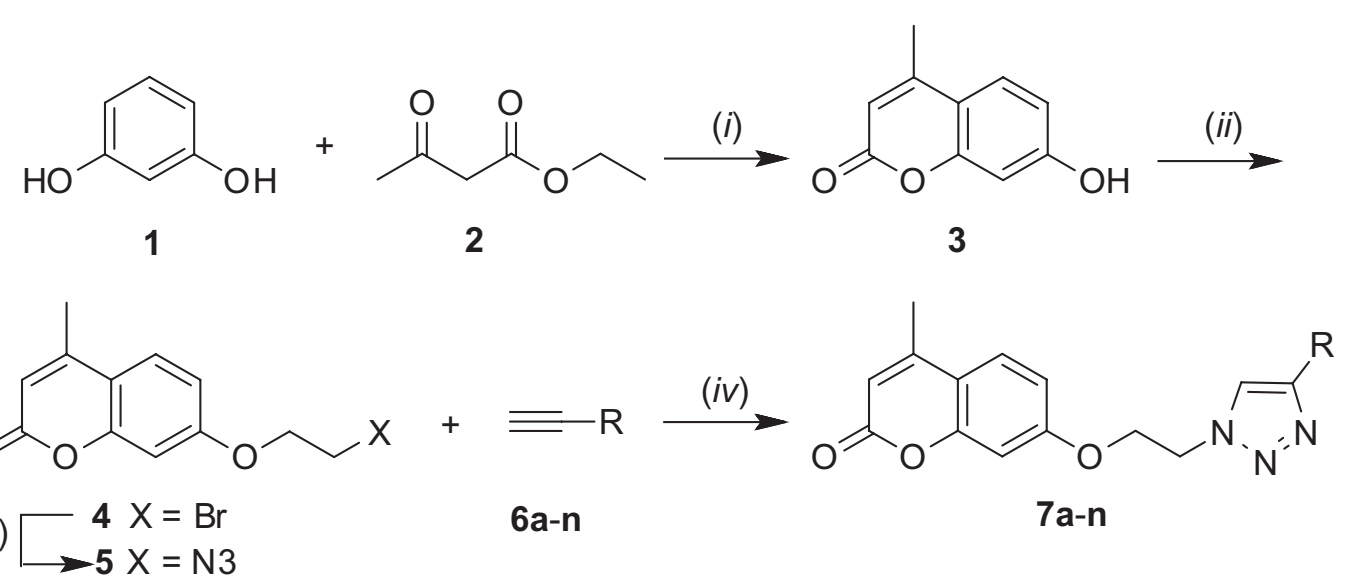

Scheme 1. Synthesis of 4-methylcoumarins/1,4-substituted 1,2,3-triazole conjugates. Reagents, conditions and yields: $(i) \mathrm{H}_{2} \mathrm{SO}_{4}$, r.t., overnight (67\%); (ii) $\mathrm{BrCH}_{2} \mathrm{CH}_{2} \mathrm{Br}, \mathrm{K}_{2} \mathrm{CO}_{3}, \mathrm{CH}_{3} \mathrm{CN}$, reflux, $6 \mathrm{~h}(52 \%)$; (iii) $\mathrm{NaN}_{3},\left(\mathrm{CH}_{3}\right)_{2} \mathrm{CO}$, reflux, $4 \mathrm{~h}(89 \%)$; and (iv) CuSO , sodium ascorbate, dimethylformamide, microwave $70{ }^{\circ} \mathrm{C}(18 \mathrm{~W}), 20-30 \min (59-91 \%)$. 
Table 1. Top five predicted targets for a 4-methylcoumarins/1,4-substituted 1,2,3-triazole conjugate using PharmMapper

\begin{tabular}{lccc}
\hline Target & PDB Code & Pharmacophore feature $^{\mathrm{a}}$ & Fit score $^{\mathrm{b}}$ \\
\hline Acetylcholinesterase & 1HBJ & 9 & 4.755 \\
PDK-1 & 2PE1 & 7 & 4.542 \\
trans-Sialidase from Trypanosoma cruzi & 1S0J & 12 & 4.519 \\
Aldose reductase & 2FZ8 & 6 & 4.458 \\
\hline
\end{tabular}

${ }^{a}$ Total number of pharmacophore features that are complementary to the pharmacophore models of the target proteins; ${ }^{b}$ pairwise alignment between the two pharmacophoric models. PDB: protein data bank; PDK-1: phosphoinositide-dependent kinase-1.

affecting the activity of butyrylcholinesterase..$^{29}$ Although few patients presented postural hypotension and dizziness after receiving ensaculin in phase I clinical trials, this drug candidate was further discontinued in phase III due to potential side effects. ${ }^{30}$<smiles>COc1ccc(N2CCN(CCCOc3cc4c(C)c(C)c(=O)oc4cc3OC)CC2)cc1</smiles>
8

Figure 2. Chemical structure of ensaculin (8, 7-methoxy-6-\{3-[4(2-methoxyphenyl)-1-piperazinyl] propoxy $\}$-3,4-dimethyl-2H-1benzopyran-2-one).

The synthesized conjugates were assayed as acetylcholinesterase inhibitors (Table 2). The most prominent compound in this series was the conjugate $\mathbf{7 g}$, which inhibited almost $60 \%$ of the enzyme activity at $200 \mu \mathrm{mol} \mathrm{L}^{-1}$. Compounds with hydrogen bond donors/ acceptor groups close to the triazole ring, e.g., compounds $\mathbf{7 a}, \mathbf{7 b}, \mathbf{7 m}$, and $\mathbf{7 n}$, also displayed intermediate inhibitory activities against $\mathrm{AChE}$.

By using molecular docking simulations, we compared the putative binding mode of the most active compound of this series, $\mathbf{7 g}$, with the crystallographic pose of the ligand suggested by the pharmacophore search in PharmMapper, the tacrine analog (Figure 3a), 4-amino-5-fluoro-2-methyl3-(3-trifluoroacetylbenzylthiomethyl)quinoline (10, PDB code $1 \mathrm{HBJ})$, and with donepezil (11, PDB code 1EVE), an acetylcholinesterase inhibitor that is clinically used to treat Alzheimer's disease.

The active site of acetylcholinesterase is composed by two subsets. The first is the catalytic subsite, which comprises residues that are essential for the hydrolase activity of the enzymes: Ser200, Glu327 and His440 (known as the catalytic triad). The second, the anionic subsite (Trp84, Tyr130, Tyr330 and Phe331), binds the quaternary trimethylammonium choline moiety of the substrate, mainly via $\pi$-cation interactions. The active site lies deep within the molecule at the base of a narrow $20 \AA$ deep gorge, lined predominantly with aromatic residues. Located at the opening of the gorge (approximately $14 \AA$ from the main active site), there is a second binding site, the peripheral anionic site, composed by the residues Tyr70, Asp72, Tyr121, Trp279 and Tyr334. The residues Trp84 in the anionic subsite and Trp279 in the peripheral anionic site have been the basis for the design of the newest generation of acetylcholinesterase inhibitors, e.g., donepezil. ${ }^{32-34}$

Compound 10 performs interactions only with residues from the catalytic and the anionic subsite of acetylcholinesterase, which compose its active site. ${ }^{35}$ This is a common feature for most of the clinically useful inhibitors of acetylcholinesterase, i.e., tacrine, rivastigmine, and galantamine, which prevent the binding of the substrate or its hydrolysis by occupying the active site with a high affinity. Donepezil, however, is the only drug that interacts with the peripheral anionic site, since its indadone moiety interacts with Trp279 whilst the benzylpiperidine fragment interacts in the catalytic site with the Trp84. ${ }^{32}$

In spite of the differences between tacrine and donepezil concerning their binding mode to the active site of acetylcholinesterase, there is no evidence that any of these drugs is superior in terms of clinical efficacy. In vitro, donepezil is approximately 40 times more potent than tacrine as an inhibitor of acetylcholinesterase; previously, we determined the $\mathrm{IC}_{50}$ of tacrine via AChE-ICER as $0.386 \pm 0.044 \mu \mathrm{mol} \mathrm{L}{ }^{-1},{ }^{15}$ while the $\mathrm{IC}_{50}$ of donepezil, calculated using immobilized AChE stationary phase, was $0.0092 \pm 0.0015 \mu \mathrm{mol} \mathrm{L}{ }^{-1} .{ }^{36}$ Clinically, donepezil has been found to be better tolerated, with less gastrointestinal side effects than rivastigmine or galantamine, while the clinical use of tacrine has been abandoned in many countries because of the high incidence of side effects, including hepatotoxicity. ${ }^{37}$ The other putative advantage of drugs that block the access of the substrate to the peripheral anionic concerns to the secondary noncholinergic functions of the enzyme (adhesion, differentiation and deposition of betaamyloid in Alzheimer's disease), which has been ascribed precisely to the peripheral anionic site. ${ }^{32,38}$ 
Table 2. Inhibition percentages of 4-methylcoumarins/1,4substituted 1,2,3-triazole conjugates against Electrophorus electricus acetylcholinesterase (AChE) immobilized onto a fused silica capillary enzyme reactor (eelAChE-ICER) in a liquid chromatography-tandem ion-trap mass spectrometer (LC-IT-MS/MS). Full structures of 4-methylumbelliferone and tacrine are shown in this table. The general structure for the corresponding R groups in the 4-methylcoumarins/1,4substituted 1,2,3-triazole conjugates is depicted in Scheme 1, structure 7

In

aThe $\mathrm{IC}_{50}$ of tacrine $\left(0.386 \pm 0.044 \mu \mathrm{mol} \mathrm{L} \mathrm{L}^{-1}\right)$ and their inhibition percentage at $200 \mu \mathrm{mol} \mathrm{L}^{-1}$ were previously determined by our research group as reported. ${ }^{16,31}$
Comparing the binding modes of $\mathbf{7 g}$ and donepezil, there is an almost perfect superposition between the phenyl ring of the $2 \mathrm{H}$-chromen-2-one core with the corresponding one from the indadone moiety from donepezil (Figure $3 b$ ), both performing $\pi-\pi$ interactions with Trp279 (Figures 3c and 3d), although this ring seems to be properly orientated to interact with Tyr121 only in donepezil 11. It was observed that the nitrogen in the piperidine from $\mathbf{1 1}$ is protonated at the physiological $\mathrm{pH}$, being able to establish a cation- $\pi$ interaction with Phe $330 .{ }^{39}$

A similar interaction is not expected to be observed in $7 \mathbf{g}$ because 1,2,3-triazoles are poorly basic in nature and, consequently, their nitrogen atoms remain non-protonated under the physiological $\mathrm{pH} .{ }^{40}$ According to the docking simulations, the triazole moiety $\mathbf{7 g}$ only interacts with one water molecule via hydrogen bonding (Figure 3d), and, therefore, makes no direct interaction with amino acid residues of AChE. Finally, while the terminal phenyl group of donepezil is directed toward Trp84 to establish $\pi-\pi$ interactions, in $\mathbf{7 g}$ the corresponding phenyl interacts with Phe330. Compound $\mathbf{7 g}$ and donepezil putatively occupy both the anionic subsite and the peripheral anionic site, the overall interactions is consistent with the fact that $\mathbf{7 g}$ is a weaker inhibitor of acetylcholinesterase than donepezil.

\section{Conclusions}

Although the most prominent compound in this series inhibited only almost $60 \%$ of the acetylcholinesterase activity at $200 \mu \mathrm{mol} \mathrm{L} \mathrm{L}^{-1}$, docking simulations suggest that this compound binds similarly to donepezil and, consequently, this compound can putatively block the secondary noncholinergic functions of the enzyme, which include adhesion, differentiation and deposition of beta-amyloid in Alzheimer's disease. Therefore, we believe that coumarin $\mathbf{7 g}$ may serve as a good starting point for further optimization of this series, but this will also require more in-deep analyses, concerning the mechanism of action of these compounds. It is now recognized that the pathogenesis of Alzheimer's disease can lead to the generation of toxic processes involving dysregulation of amyloid precursor protein metabolism, inflammation, oxidative stress, apoptosis, proteosome inhibition, mitochondrial dysfunction, imbalances in antioxidant production and glutamate excitotoxicity, amongst others. ${ }^{41}$ Therefore, anti-inflammatory and antioxidant actions, which are typically observed for natural coumarins, ${ }^{8}$ can also be explored in 4-methylcoumarins/1,2,3triazoles conjugates to yield multitarget ligands in the search of new compounds for the treatment of Alzheimer's disease. 
<smiles>Cc1nc2cccc(F)c2c(N)c1CSCc1cccc(C(=O)C(F)(F)F)c1</smiles><smiles>COc1cc2c(cc1OC)C(=O)C(CC1CCN(Cc3ccccc3)CC1)C2</smiles><smiles>Cc1cc(=O)oc2cc(OCCn3cc(CCc4ccccc4)nn3)ccc12</smiles>

(a)
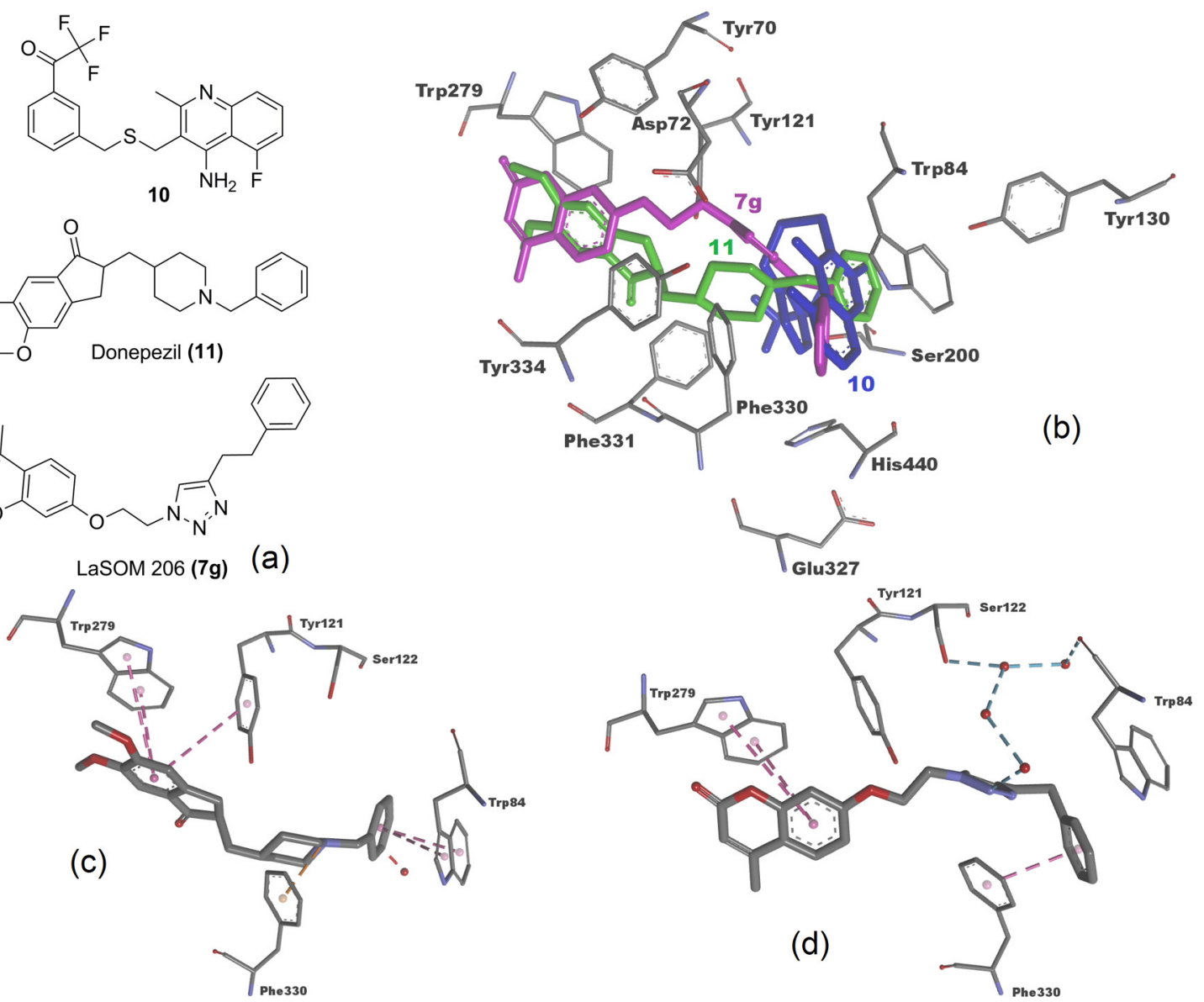

Figure 3. (a) Two-dimensional structure representations of 4-amino-5-fluoro-2-methyl-3-(3-trifluoroacetylbenzylthiomethyl)quinoline (10), donepezil (11) and $\mathbf{7 g}$; (b) comparison of the binding modes of $\mathbf{1 0}, \mathbf{1 1}$ and $\mathbf{7 g}$; (c) crystallographic structure of donepezil within the acetylcholinesterase binding cleft, highlighting the main intermolecular interactions: phenyl (indadone)/Trp279 ( $\pi-\pi ; 4.08,5.30 \AA$ ), nitrogen (piperidine)/Phe330 (cation- $\pi$, $3.14 \AA$ ), phenyl/Trp84 $(\pi-\pi ; 4.04,4.64 \AA)$; and (d) docked binding mode of $\mathbf{7 g}$ within the acetylcholinesterase binding cleft, highlighting the main intermolecular interactions: phenyl ( $2 H$-chromen-2-one)/Trp279 $(\pi-\pi ; 4.66,5.61 \AA)$, nitrogen (1,2,3-triazole)/water (H bond; $2.58 \AA$ ), phenyl/Phe330 ( $\pi-\pi ; 3.97 \AA)$.

\section{Supplementary Information}

Supplementary information is available free of charge at http://jbcs.sbq.org.br.

\section{Acknowledgments}

The authors thank the Brazilian funding agencies Coordenação de Aperfeiçoamento de Pessoal de Nível Superior (CAPES), Conselho Nacional de Desenvolvimento Científico e Tecnológico (CNPq), Fundação de Amparo à Pesquisa do Estado do Rio Grande do Sul (FAPERGS), Fundação de Amparo à Pesquisa do Estado de São Paulo (FAPESP) and Instituto Nacional de Ciência e Tecnologia para Inovação Farmacêutica (INCT-IF), for the financial support. The authors are thankful to Prof Saulo F. de Andrade (PPGCF/UFRGS) and Prof Francisco P. dos Santos (IQ/UFRGS), for the support on the NMR analyses; and to Maristela C. da S. Piedade (LaSOM/UFRGS) and
Maribete H. Holzschuh (PPGCF/UFRGS), for the technical support.

\section{References}

1. Gómez-Outes A.; Suárez-Gea, M. L.; Calvo-Rojas, G.; Lecumberri, R.; Rocha, E.; Pozo-Hernández, C.; TerleiraFernández, A. I.; Vargas-Castrillón, E.; Curr. Drug Discovery Technol. 2012, 9, 83.

2. Matos, M. J.; Viña, D.; Vazquez-Rodriguez, S.; Uriarte, E.; Santana, L.; Curr. Top. Med. Chem. (Sharjah, United Arab Emirates) 2012, 12, 2210.

3. Vianna, D. R.; Bubols, G.; Meirelles, G.; Silva, B.; Rocha, A.; Lanznaster, M.; Monserrat, J. S.; Garcia, S. C.; von Poser, G.; Eifler-Lima, V. L.; Int. J. Mol. Sci. 2012, 13, 7260.

4. Vianna, D. R.; Hamerski, L.; Figueiró, F.; Bernardi, A.; Visentin, L.; Teixeira, H.; Pires, E.; Eifler-Lima, V. L.; Salbego, C.; Batasttini, A. M.; von Poser, G.; Pinto, A.; Eur. J. Med. Chem. 2012, 57, 268. 
5. Vianna, D. R.; Ruschel, L.; Dietrich, F.; Figueiró, F.; Morrone, F. B.; Canto, R. F. S.; Corvello, F.; Velho, A.; Crestani, A.; Teixeira, H.; von Poser, G. L.; Battastini, A. M. O.; Eifler-Lima, V. L.; MedChemComm 2015, 6, 905.

6. Matos, M. J.; Vazquez-Rodriguez, S.; Santana, L.; Uriarte, E.; Fuentes-Edfuf, C.; Santos, Y.; Muñoz-Crego, A.; Med. Chem. (Sharjah, United Arab Emirates) 2012, 8, 1140.

7. Evans, B. E.; Rittle, K. E.; Bock, M. G.; DiPardo, R. M.; Freidinger, R. M.; Whitter, W. L.; Lundell, G. F.; Veber, D. F.; Anderson, P. S.; J. Med. Chem. 1988, 31, 2235.

8. Torres, F. C.; Brucker, N.; Andrade, S. F.; Kawano, D. F.; Garcia, S. C.; Poser, G. L. V.; Eifler-Lima, V. L.; Curr. Top. Med. Chem. (Sharjah, United Arab Emirates) 2014, 14, 2600.

9. Ritchie, T. J.; Macdonald, S. J.; Drug Discovery Today 2009 , $14,1011$.

10. Zhou, C. H.; Wang, Y.; Curr. Med. Chem. 2012, 19, 239.

11. Aragão-Leoneti, V.; Campo, V. L.; Gomes, A. S.; Field, R. A.; Carvalho, I.; Tetrahedron 2010, 66, 9475.

12. Armarego, W. L. E.; Chai, C. L. L.; Purification of Laboratory Chemicals, $5^{\text {th }}$ ed.; Butterworth-Heinemann: Burlington, 2003.

13. Russell, A.; Frye, J. R.; Org. Synth. 1941, 21, 22.

14. Duan, Y. C.; Ma, Y. C.; Zhang, E.; Shi, X. J.; Wang, M. M.; Ye, X. W.; Liu, H. M.; Eur. J. Med. Chem. 2013, 62, 11.

15. Carvalho, I.; Andrade, P.; Campo, V. L.; Guedes, P. M.; SestiCosta, R.; Silva, J. S.; Schenkman, S.; Dedola, S.; Hill, L.; Rejzek, M.; Nepogodiev, S. A.; Field, R. A.; Bioorg. Med. Chem. 2010, 18, 2412.

16. Vanzolini, K. L.; Vieira, L. C. C.; Correa, A. G.; Cardoso, C. L.; Cass, Q. B.; J. Med. Chem. 2013, 56, 2038.

17. http://www.molecular-networks.com/online_demos/corina_ demo accessed in January 2016.

18. Gasteiger, J.; Rudolph, C.; Sadowski, J.; Tetrahedron Comput. Methodol. 1990, 3, 537.

19. Thompson, M. A.; Proc. ACS Meet. 2004, 42, 172. Available at http://www.fis.unam.mx/ ramon/CursoDF/Material\%20 Didactico/ArgusLab/Papiros/Docking_With_ArgusLab.ppt accessed in January 2016.

20. http://59.78.96.61/pharmmapper/ accessed in January 2016.

21. Liu, X.; Ouyang, S.; Yu, B.; Liu, Y.; Huang, K.; Gong, J.; Zheng, S.; Li, Z.; Li, H.; Jiang, H.; Nucleic Acids Res. 2010, 38, W609.

22. http://lorentz.immstr.pasteur.fr/pdb/frozen_submission.php accessed in January 2016.

23. http://nbcr-222.ucsd.edu/pdb2pqr_2.0.0/, accessed in January 2016.

24. http://www.ccdc.cam.ac.uk/solutions/csd-discovery/ components/gold/ accessed in January 2016.
25. Young, D. C.; Computational Drug Design: a Guide for Computational and Medicinal Chemists; John Wiley \& Sons: Oxford, 2009.

26. Rodrigues, R. P.; Andrade, S. F.; Mantoani, S. P.; Eifler-Lima, V. L.; Silva, V. B.; Kawano, D. F.; J. Chem. Educ. 2015, 92, 827.

27. Discovery Studio Visualizer Version 4.0; Dassault Systèmes, USA, 2013.

28. Anand, P.; Singh, B.; Singh, N.; Bioorg. Med. Chem. 2012, 20, 1175.

29. Hoerr, R.; Noeldner, M.; CNS Drug Rev. 2002, 8, 143.

30. Lindner, M. D.; Pharmacol. Ther. 2007, 115, 148.

31. Sangi, D. P.; Monteiro, J. L.; Vanzolini, K. L.; Cass, Q. B.; Paixão, M. W.; Corrêa, A. G.; J. Braz. Chem. Soc. 2014, 25, 887.

32. Castro, A.; Martinez, A.; Mini-Rev. Med. Chem. 2001, 1, 267.

33. Johnson, G.; Moore, S. W.; Curr. Pharm. Des. 2006, 12, 217.

34. Silva, V. B.; Andrade, P.; Kawano, D. F.; Morais, P. A.; Almeida, J. R.; Carvalho, I.; Taft, C. A.; Silva, C. H. T. P.; Future Med. Chem. 2011, 3, 947.

35. Doucet-Personeni, C.; Bentley, P. D.; Fletcher, R. J.; Kinkaid, A.; Kryger, G.; Pirard, B.; Taylor, A.; Taylor, R.; Taylor, J.; Viner, R.; Silman, I.; Sussman, J. L.; Greenblatt, H. M.; Lewis, T.; J. Med. Chem. 2001, 44, 3203.

36. Andrisano, V.; Bartolini, M.; Gotti, R.; Cavrini, V.; Felix, G.; J. Chromatogr. B: Biomed. Sci. Appl. 2001, 753, 375.

37. Colović, M. B.; Krstić, D. Z.; Lazarević-Pašti, T. D.; Bondžić, A. M.; Vasić, V. M.; Curr. Neuropharmacol. 2013, 11, 315.

38. Johnson, G.; Moore, S. W.; Biochem. Biophys. Res. Commun. 1999, $258,758$.

39. Kryger, G.; Silman, I.; Sussman, J. L.; Structure (Oxford, U. K.) 1999, 7, 297.

40. Tripathi, R. P.; Dwivedi, P.; Sharma, A.; Kushwaha, D.; Tiwari, V. K. In Click Chemistry in Glycoscience: New Developments and Strategies; Witczak, Z. J.; Bielski, R., eds.; John Wiley \& Sons: Oxford, 2013, ch. 12.

41. Walker, D.; Lue, L.-F.; Curr. Neuropharmacol. 2007, 5, 232.

Submitted: November 10, 2015

Published online: February 1, 2016

FAPERGS/CAPES and FAPESP have sponsored the publication of this article. 